\title{
Experiment and Smooth Particle Hydrodynamics Simulation for the Wear Characteristics of Single Diamond Grit Scratching on Sapphire
}

\author{
Haiyong WU \\ College of Mechanical Engineering, Zhangzhou Institute of Technology, Zhangzhou, 363000, Fujian Province, China. \\ E-mail: haiyongwu@126.com \\ College of Mechanical Engineering and Automation, Huaqiao University, Xiamen, Fujian Province, 361021, China \\ Post-Doctoral Research Center, Fujian Longxi Bearing (Group) Corp., LTD., Zhangzhou, 363000, Fujian Province, \\ China
}

crossref http://dx.doi.org/10.5755/j02.mech.27370

\section{Introduction}

Sapphire is widely used in semiconductor lighting, large-scale integrated circuits, high-intensity laser windows and other fields [1]. Due to the extremely high hardness and hard machinability of sapphire, abrasive single crystal diamond (SCD) grits are widely employed in the wire sawing, grinding, lapping and polishing process of sapphire. Although the SCD grit is the hardest material in nature, it presents to be rubdown, micro-fractured, macrofractured and breaking-off during the machining process of sapphire [2].

The wear characteristic of SCD grit has a signifycant influence on the machining performance of SCD tools and surface topography of sapphire [3]. The wear progress of SCD grit could be evaluated by simulations. And mesh method and meshless method are the two main simulation methods $[4,5]$.

The mesh method is principally based on finite element method (FEM). In the previous studies, SCD grit is mostly regarded as a rigid body, and that leads to the wear characteristics cannot be well demonstrated factually [6].

The messless method is mainly based on molecular dynamics (MD) method and smooth particle hydrodynamics (SPH) method [7]. The MD method is primarily carried out in nano-scale simulation for the ultra-precision machining of large SCD, and the SPH method is mainly employed in micro-scale simulation for abrasive SCD grit [8]. However, the abrasive SCD grit is almost regarded as an elastic body in the post SPH simulation, and that lead to the simulation results are slightly different from actual wear situation [9].

This paper attempts to present and illustrate the wear characteristics of a single abrasive SCD grit scratching on sapphire with SPH simulation and experiment. The Steinberg constitutive equation and Grüneisen state equation (SG equation) are employed in the SPH simulation. The wear morphology, wear volume and scratching forces are measured and tracked. With the combination analysis of SPH simulation and experiment, this paper aims to reveal the wear mechanism of single SCD grit scratching on sapphire.

\section{SPH method and model}

\subsection{SPH method}

SPH method is a dynamic problem solver with meshless, adaptive, stable and Lagrangian properties. The core of SPH method is interpolation theory. The solution of SPH method is mainly based on the partial differential equations of density, velocity and energy. For SPH method, a series of arbitrarily distributed particles are employed to represent the problem domain, and integral representation is employed to approximate the field function. The particles are used to further approximate the kernel function equation and carried out to approximation process in each time step. A series of time-dependent discrete ordinary differential equations can be obtained by applying the sub kernel function method to the field function correlation terms of partial differential equations [10]. The particle approximation function in the SPH method can be obtained by:

$$
\Pi^{h} f(x)=\int f(y) W(x-y, h) d y
$$

where: $W$ is a smoothing kernel function; $h$ is the smooth length of the affected area of defined kernel function $W$. The kernel function could be defined by auxiliary function $\theta$ :

$$
W(x, h)=\frac{1}{h(x)^{d}} \theta(x),
$$

where: $d$ is the spatial dimension; $h$ is the smooth length and the auxiliary function $\theta$ can be expressed by:

$$
\theta(u)=C \times\left\{\begin{array}{l}
1-1.5 u^{2}+0.75 u^{3},|u| \leq 1 \\
0.25(2-u)^{3}, 1 \triangleleft u \mid \leq 2 \\
0,|u|>2
\end{array}\right.
$$

where: $C$ is normalization constant. The field function is expressed in the form of pairs of particles. The SPH simulation of this paper is based on the above equations.

\subsection{SPH model}

The physical model of SPH simulation is based on the actual abrasive SCD grit (Fig. 1, a). The physical geometrical model of SCD grit can be established by PROE software (Fig. 1, b).

The grit-work piece contact area is selected as research object (Fig. 1, c). The SCD grit is point contact with work piece, and the $\{100\}$ crystal plane of SCD grit is in the front of the scratching direction. 
The grit-work piece contact part of SCD grit is meshed and transformed to SPH particles by LS-PrePost software (V4.0) and LS-DYNA software (V9.71_R7.0 version, Fig. 2, a). The size of each SPH particle is $0.005 \mathrm{~mm}$, and the total number of the SPH particles is 3511 . The physical model of sapphire $(2 \times 0.15 \times 0.06 \mathrm{~mm})$ is modelled by FEM, and its element total number and minimum size is 24120 and $0.01 \mathrm{~mm}$ respectively (Fig. 2, b).

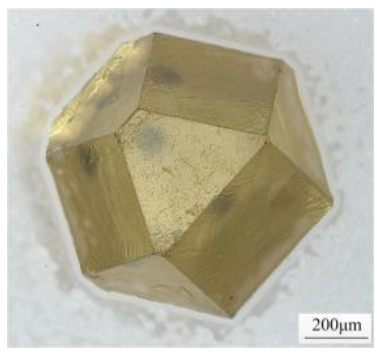

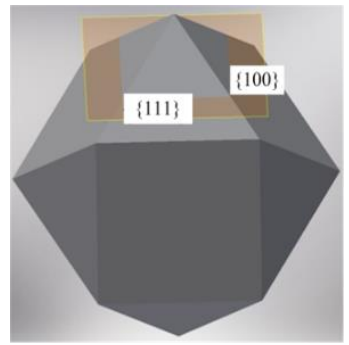

b

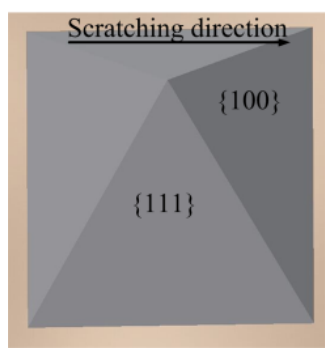

Fig. 1 The actual abrasive cub-octahedral SCD grit (a), its physical model (b) and contact part (c)

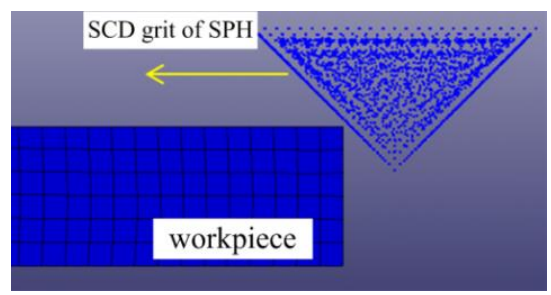

a

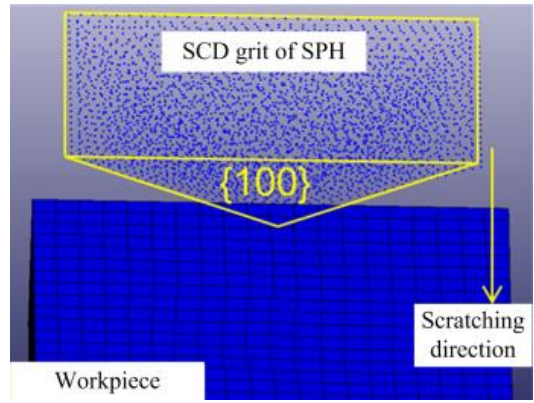

b

Fig. 2 The physical model (a) and scratching direction schematic diagram (b)

The Steinberg constitutive equation, which is employed in the material model of SCD grit, is described as:

$$
\begin{aligned}
& Y=Y_{0}\left[1+\beta\left(\xi+\xi_{i}\right)\right]^{n} \times \\
& \times\left[1+\frac{Y_{p}^{\prime}}{Y_{0}} \frac{p}{\eta^{1 / 3}}+\frac{G_{T}^{\prime}}{G_{0}}(T-300)\right] .
\end{aligned}
$$

where: $G$ is the shear modulus; $G_{0}$ is the shear modulus under normal temperature and pressure; $Y$ is the yield strength; $Y_{0}$ is the initial yield strength of the highest strain rate or lowest temperature test; $p$ is the pressure; $T$ is the temperature; $\eta=V_{0} / V$ is the compression ratio; $\beta, n$ are the strain hardening parameters; $\xi$ is the plastic strain; $\xi_{0}$ is the initial plastic strain; $G_{p}^{\prime} ; G_{T}^{\prime}$ and $Y_{p}^{\prime}$ are the first-order partial derivatives of $G$ and $Y$ with respect to the pressure $p$ or temperature $T ; Y_{\max }$ is the maximum allowable strain hardening; $T_{m}$ is the melting temperature related to pressure.

Meanwhile, the Grüneisen state equation is employed in the simulation, and it could be expressed as:

$$
p-p_{0}=\frac{\gamma}{\varphi}\left(E-E_{0}\right),
$$

where: $\gamma$ is the Grüneisen parameter; $p_{0}, E_{0}$ are cold pressure and cold energy at $0 \mathrm{~K}$ temperature; pressure $p$ and internal energy $E$ are functions of specific volume $\varphi$ and temperature $T$; cold pressure $p_{0}$ and cold energy $E_{0}$ are only related to the specific heat capacity of the object $\varphi$.

The parameters of Steinberg constitutive equation for SCD grit are shown in Table 1. Johnson-Holmquist Ceramic (JHC) constitutive model is employed for sapphire, and the model can be expressed as:

$$
\begin{aligned}
& \sigma_{\mathrm{i}}^{*}=A \quad\left(P^{*}+T^{*}\right)^{N}\left[1+C \ln \left(\dot{\varepsilon} / \varepsilon_{0}\right)\right], \\
& \sigma_{\mathrm{f}}^{*}=B\left(P^{*}\right)^{M}\left[1+C \ln \left(\dot{\varepsilon} / \varepsilon_{0}\right)\right], \\
& \varepsilon_{p}^{f}=D_{l}\left(P^{*}+T^{*}\right) D_{2},
\end{aligned}
$$

where: $\sigma_{i}^{*}$ and $\sigma_{f}^{*}$ are dimensionless equivalent stress of undamaged material and damaged material; $A, B, C, M, N$ and $T$ are material constant of the strength model; $P^{*}$ is dimensionless hydrostatic pressure and $P^{*}=P / P_{H E L} ; T^{*}=$ $=T / P_{\mathrm{HEL}} ; T$ is the maximum hydrostatic tension that the material can withstand. The dimensionless strain rate is equal to the ratio of the true strain rate $\varepsilon$ to the reference strain rate $\varepsilon ; P_{H E L}$ is the hydrostatic pressure tensor of the material in the Hugoniot elastic limit (HEL) state; $D_{1}$ and $D_{2}$ are the material constants in damage model.

Table 1

The parameters of Steinberg constitutive equation for SCD grit

\begin{tabular}{|c|c|c|c|c|c|c|c|}
\hline Parameter & $\begin{array}{c}Y_{0}, \\
\mathrm{GPa}\end{array}$ & $\begin{array}{c}\beta, \\
-\end{array}$ & $\begin{array}{c}\xi_{0}, \\
-\end{array}$ & $\begin{array}{c}n, \\
-\end{array}$ & $\begin{array}{c}\rho_{0}, \\
\mathrm{~g} / \mathrm{cm}^{3}\end{array}$ & $\begin{array}{c}Y_{\max }, \\
\mathrm{GPa}\end{array}$ & $\eta,-$ \\
\hline Value & 90 & 0 & 0 & 0.27 & 3.51 & 1000 & 0.88 \\
\hline
\end{tabular}

The equation of state of the material in the $\mathrm{JHC}$ constitutive model can be expressed as:

$$
P=K_{1} \mu+K_{2} \mu^{2}+K_{3} \mu^{3},
$$

where: $P$ is hydrostatic pressure; $K_{1}$ is material bulk modulus; $K_{2}, K_{3}$ are material constants; $\mu=\rho / \rho_{0}-1 ; . \rho$ is the instantaneous density when the material is compressed; $\rho_{0}$ is the initial density of the material.

The SPH model parameters are shown in Table 2 
$[11,12]$. The simulation scratching depth is $0.02 \mathrm{~mm}$ and scratching speed is $4 \mathrm{~m} / \mathrm{s}$, they are the same as the experimental parameters. The scratching length of sapphire model is $2 \mathrm{~mm}$, the total simulation time is $0.5 \mathrm{~ms}$ and calculation step is $5 \times 10^{-4} \mathrm{~ms}$.

Table 2

The model parameters of SPH simulation

\begin{tabular}{|c|c|c|c|}
\hline Model parameter & Value & Model parameter & Value \\
\hline$A$ & 0.93 & HEL Strength, GPa & 2.0 \\
\hline$B$ & 0.31 & Ref Strain Rate (EPSI) & 1.0 \\
\hline$C$ & 0.0 & $D_{1}$ & 0.005 \\
\hline$M$ & 0.6 & $D_{2}$ & 1.0 \\
\hline$N$ & 0.6 & $K_{1}, \mathrm{GPa}$ (Bulk modulus) & 130.95 \\
\hline HEL, GPa & 2.79 & $K_{2}, \mathrm{GPa}$ & 0 \\
\hline $\begin{array}{c}\text { HEL Pressure, } \\
\text { GPa }\end{array}$ & 1.46 & $K_{3}, \mathrm{GPa}$ & 0 \\
\hline HEL Vol. Strain & 0.0112 & Beta & 1.0 \\
\hline
\end{tabular}

\section{Experimental procedure}

The scratching experiment was carried out by a precision scratch test setup which performance parameters are shown in Table 3. Common abrasive SCD grits (Element Six SDB 1125, and particle size is $0.6-0.85 \mathrm{~mm}$ ) were brazed onto a stainless steel blind nut with $\mathrm{NiCrBSi}$ alloy (Fig. 3). The blind nut was fixed on an aluminium alloy clamp which was mounted onto a Kistler 9119AA2 dynamometer. A Mitutoyo micro-meter head is used to control the scratching depth. The SCD grit could reciprocate by a linear motor. A polished sapphire wafer, whose properties are shown in Table 4, was stuck onto a finish machined aluminium plate. The aluminium plate was fixed to the machine spindle with threaded connection.

Table 3

The performance parameters of precision scratch test setup

\begin{tabular}{|c|c|c|c|}
\hline $\begin{array}{c}\text { Spindle } \\
\text { maximum } \\
\text { speed, } \mathrm{rpm}\end{array}$ & $\begin{array}{c}\text { Spindle rotat- } \\
\text { ing accuracy, } \\
\mu \mathrm{m}\end{array}$ & $\begin{array}{c}\text { Face runout of } \\
\text { sample table, } \\
\mu \mathrm{m}\end{array}$ & $\begin{array}{c}\text { Scratch accuracy, } \\
\mu \mathrm{m}\end{array}$ \\
\hline 12000 & 1 & 2 & 2 \\
\hline
\end{tabular}

Table 4

Properties of sapphire employed in the experiment

\begin{tabular}{|c|c|c|c|c|c|}
\hline $\begin{array}{c}\text { Hardness, } \\
\mathrm{GPa}\end{array}$ & $\begin{array}{c}\text { Melting tempera- } \\
\text { ture, }{ }^{\circ} \mathrm{C}\end{array}$ & $\begin{array}{c}\text { Boiling } \\
\text { point, }{ }^{\circ} \mathrm{C}\end{array}$ & $\begin{array}{c}\text { Tensile } \\
\text { strengt, } \\
\mathrm{MPa}\end{array}$ & $\begin{array}{c}\text { Shear } \\
\text { modulus, } \\
\mathrm{GPa}\end{array}$ & $\begin{array}{c}\mathrm{Ra}, \\
\mathrm{nm}\end{array}$ \\
\hline 20 & 2045 & 3000 & 200 & 90.16 & 10 \\
\hline
\end{tabular}

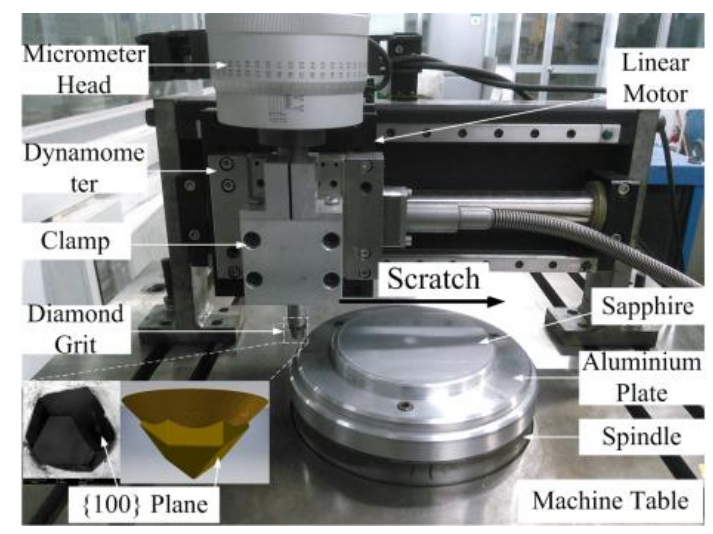

Fig. 3 The scratching experiment setup
SEM images (by FEI Phenom prox), wear volume Qd (by Carl Zeiss LSM700) and workpiece material removal volume $Q w$ (by ZYGO NewView 7300) were metro-logically measured at a regular scratching distance interval $(20 \mathrm{~mm})$. The SCD grit scratches on sapphire until it is complete wear in the experiment. The total scratching length can be calculated by $l w=w \times 20 \mathrm{~mm}, w$ is the total scratching intervals. The experimental scratching depth and scratching speed are the same with the SPH simulation parameters.

\section{Results}

\subsection{Wear morphology of SPH simulation}

The simulation result is shown in Fig. 4. It can be observed that the sapphire material is brittle removed on both sides of the scratch groove.

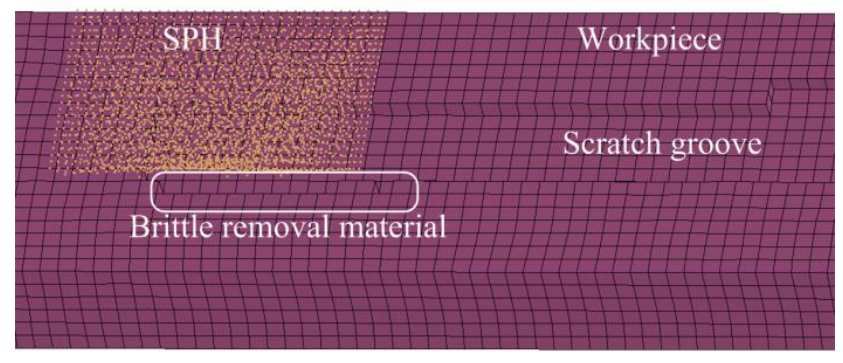

Fig. 4 The SPH particles of SCD grit scratch on sapphire workpiece

The SPH particles, which deviate from the body range of SCD grit, can be regarded as wear. The wear volume could be obtained with calculating the total number of deviated SPH particles by the LS-Prepost software automatically. The wear progress of SPH particles are shown in Fig. 5.

The $\{100\}$ crystal plane is in the front of scratching direction. At initial stage at $t=0.1 \mathrm{~ms}$ (Fig. 5, a), the stress of front SPH particles increase obviously. The SPH particles deviate and form a local small gap. With the increasing of scratching time, the number of deviating SPH particles increases and the wear area enlarges gradually. It could be observed that a small amount of the rear SPH particles are wear at $t=0.3 \mathrm{~ms}$ (Fig. 5, b). With the increasing of workpiece material removal volume (Fig. 5, c), the deviating SPH particles both at the front and the rear of scratching direction increases gradually, and the wear area enlarges continuously.

\subsection{Wear morphology of experiment}

The morphology of sapphire is shown in Fig. 6. It can be observed that the scratch on sapphire is formed by brittle fracture (Fig. 6, a) and vary in depth (Fig. 6, b). The sapphire removal characteristic is similar to the simulation results (Fig. 4). The average cross sectional area $S_{w}$ of the sapphire scratch can be calculated with five cross curve area. Therefore, the material removal volume $Q w$ could be defined as:

$$
Q w=S_{w} \times l_{w} .
$$




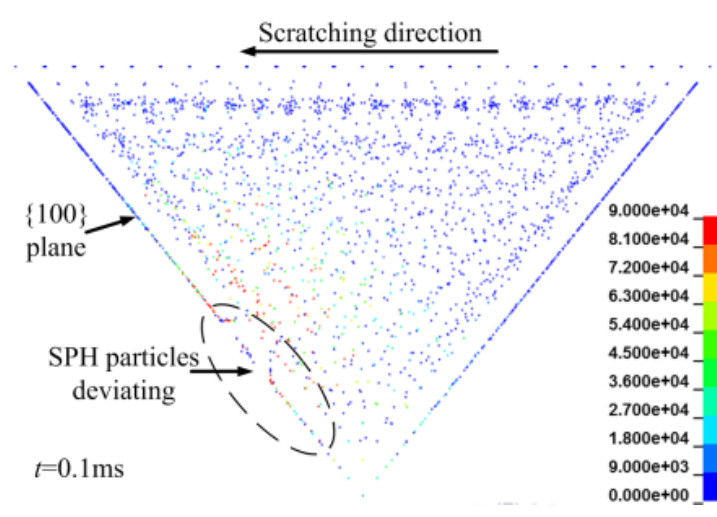

a

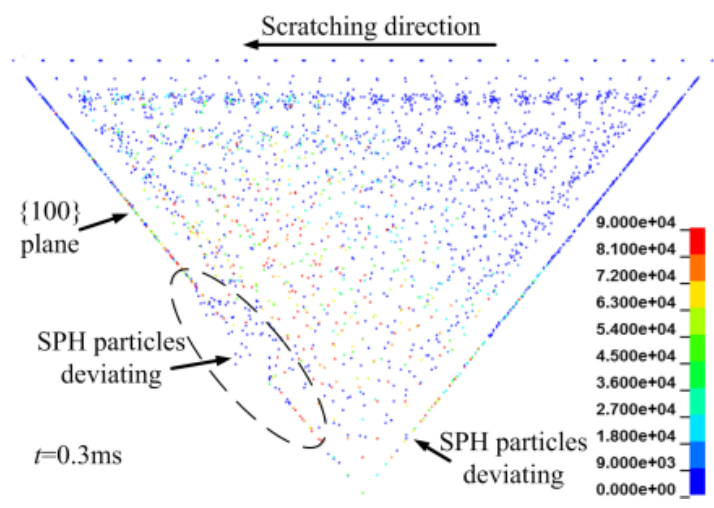

b

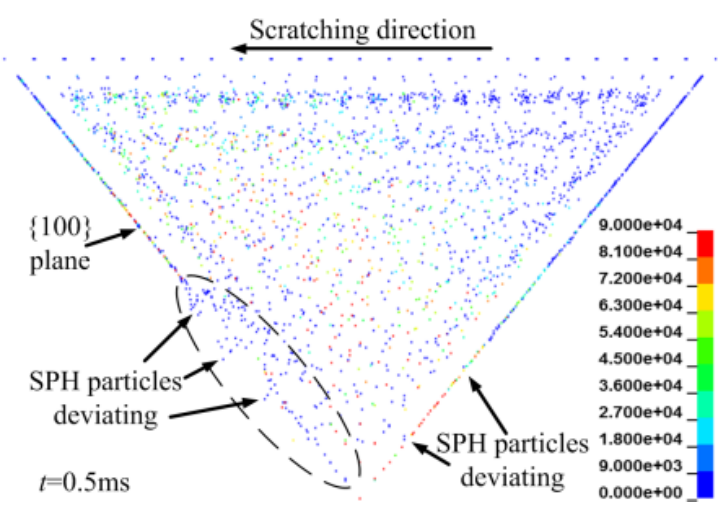

C

Fig. 5 The wear progress of SCD grit in SPH simulation at different stages: $t=0.1 \mathrm{~ms}$ (a), $t=0.3 \mathrm{~ms}$ (b) and $t=$ $=0.5 \mathrm{~ms}(\mathrm{c})$

The SEM morphologies at different wear stages are shown in Fig. 7. It can be observed that the gritworkpiece contact area of SCD grit is damaged obviously (Fig. 7, b). A penetrating crack is produced across the SCD grit. Several cracks and fractures appear in the front $\{100\}$ plane of SCD grit, and flaky cleavages appear in the rear $\{111\}$ plane. With the increasing of workpiece material removal volume, the wear state becomes more severe (Fig. 7, c). The front of SCD grit is almost pulled out, and more cleavage fractures and cracks are produced at the rear of SCD grit. Finally, the exposure part of SCD grit breaks off from the tool substrate integrally (Fig. 7, d).

\subsection{Wear volume}

The wear volume variations of SPH simulation and experiment are shown in Fig. 8. The SPH particles,

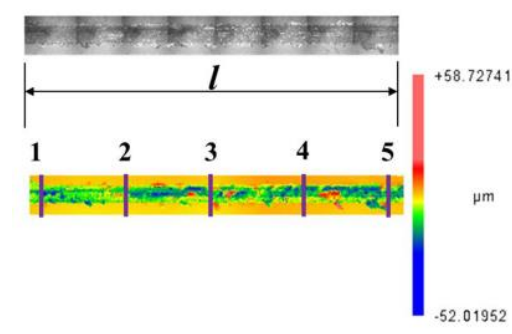

$\mathrm{a}$

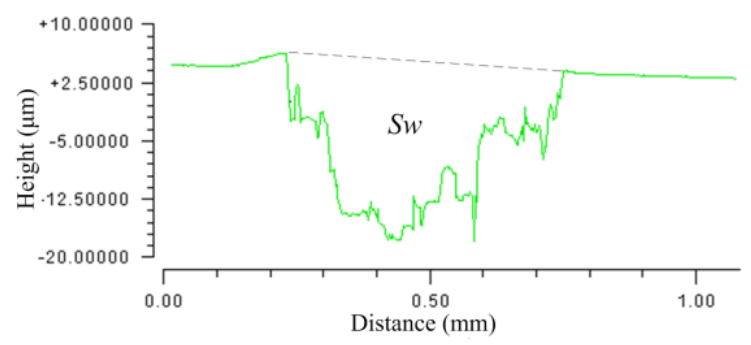

$\mathrm{b}$

Fig. 6 The scratch morphology of sapphire (a) and its cross section curve (b)

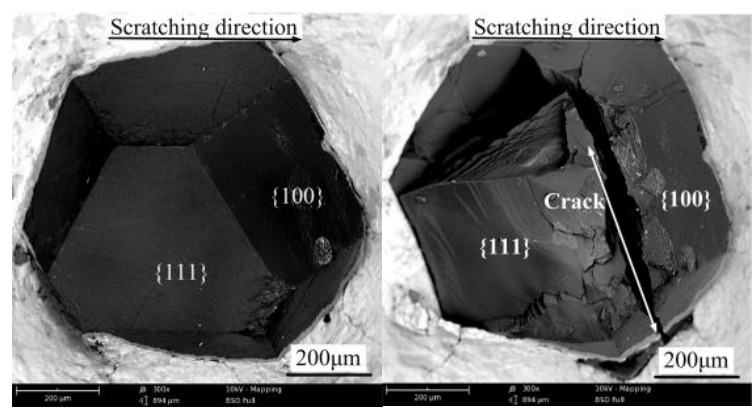

a

b

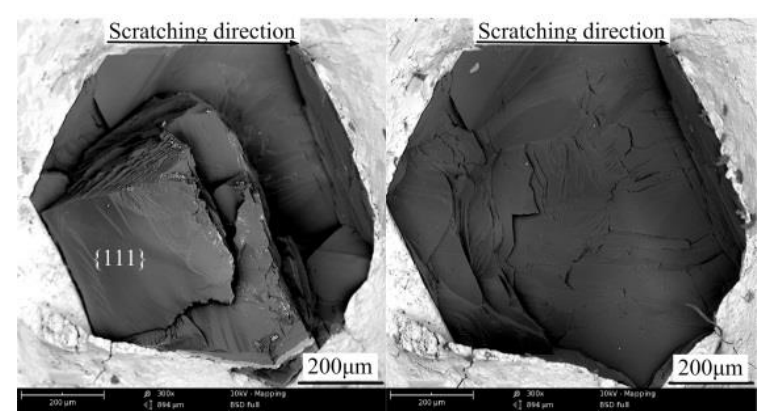

c

d

Fig. 7 The wear morphology of SCD grit at different stages: $Q w=0 \mathrm{~mm}^{3}$ (a), $Q w=0.18 \mathrm{~mm}^{3}$ (b), $Q w=$ $=0.27 \mathrm{~mm}^{3}$ (c) and $Q w=0.36 \mathrm{~mm}^{3}(\mathrm{~d})$

which are deviating from the SCD grit body range, are regarded as the wear particles. The wear volume $Q_{N}$ of SPH particles could be expressed as:

$$
Q_{N}=\frac{Q_{n}}{Q_{t}}
$$

where: $Q_{n}$ is the number of deviating particles; $Q_{t}$ is the total number of SPH particles. The final experiment scratching length is $80 \mathrm{~mm}$ and the wear volume can be calculated by Eq. (10). The wear volume variation trend of both SPH simulation (Fig. 8, a) and experiment (Fig. 8, b) increase linearly with the increasing work piece removal 
volume similarly. The wear volume curves of SPH simulation and experiment could be well linear fitted by $Q_{N}=$ $=0.17316 Q w+0.0732$ and $Q d=0.1943 Q w-0.00638$ respectively. The slope of the two fitting line could be regarded as the wear rate, and the wear rate of the simulation and experiment are approximately the same.

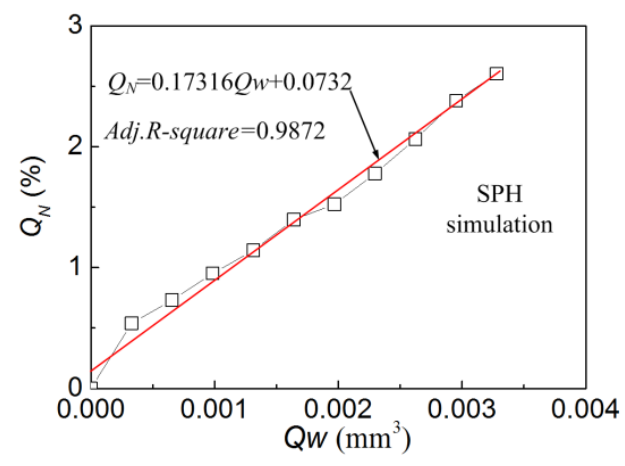

a

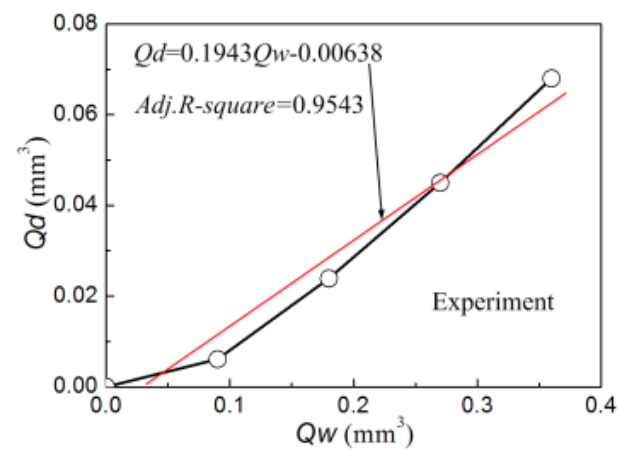

b

Fig. 8 Wear volume variations of SCD grit in SPH simulation (a) and experiment (b) respectively

\subsection{Scratching force}

The scratching tangential force $F t$ and normal force $F n$ for SPH simulation and experiment are shown in Fig. 9. Both the Ft and Fn increase quickly in the beginning and fluctuate in certain ranges (approximately 25 $65 \mathrm{~N}$ for $F n$ and $5-18 \mathrm{~N}$ for $F t$ ) during the simulation (Fig. 9, a).

It can be observed that both experimental $F t$ and Fn (Fig. 9, b) exhibit to fluctuate in a certain major range due to the brittle removal volume of sapphire (Fig. 6, a). In order to eliminate the fluctuation and noise of scratching force signals, Savizkg-Golag algorithm is employed to smooth the scratching forces (Fig. 9, c). The scratching forces fluctuate in a smaller range (approximately 28 $53 \mathrm{~N}$ for $F n$ and $9-36 \mathrm{~N}$ for $F t$ ). For the scratching forces of SPH simulation (Fig. 9, a) and experiment (Fig. 9, c), their variation trends are similar and value ranges are approximately equal. It could be observed that both the $F t$ and $F n$ exhibit to decrease with the increasing of workpiece removal volumes (Fig. 9, d). That is because the gritworkpiece contact area decreases during the wear progress (Fig. 7) and fewer workpiece material volumes are removed.

\section{Discussion}

The SPH simulation results show that the wear progress of the SCD grit is closely related to the stress state during the scratching process, it is also demonstrated by the experimental results of force variation (Fig. 9) and morphology progress (Figs. 7 and 8). Meanwhile, it is also demonstrated in the post studies [13] that the stress distribution during the scratching process has a significant influence on the wear characteristics of SCD grit. Thus, the stress theoretical model should be established to the further analyse of the wear mechanism of SCD grit.

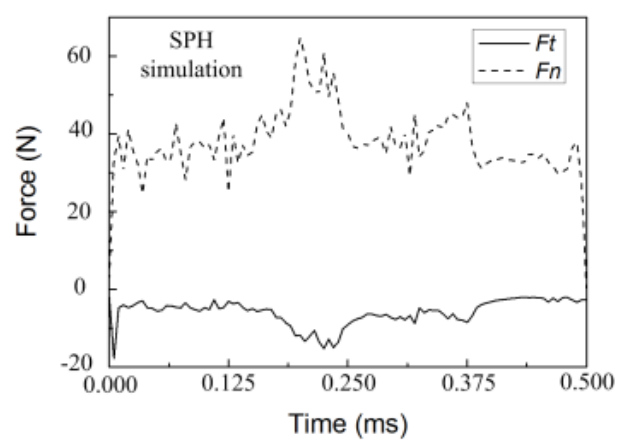

a

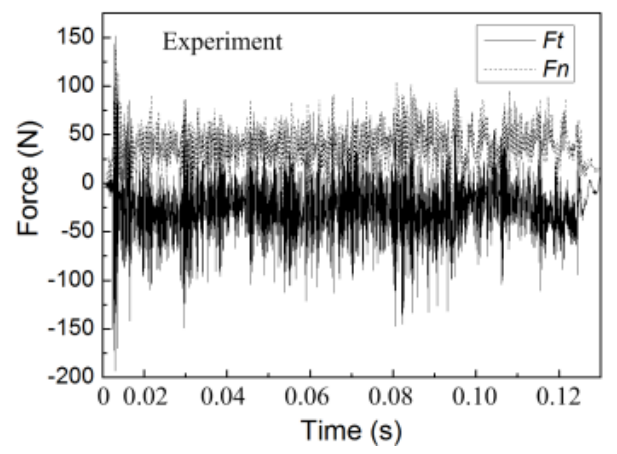

b

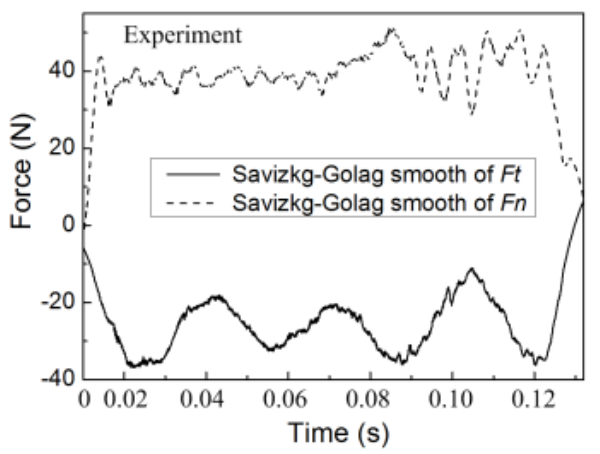

c

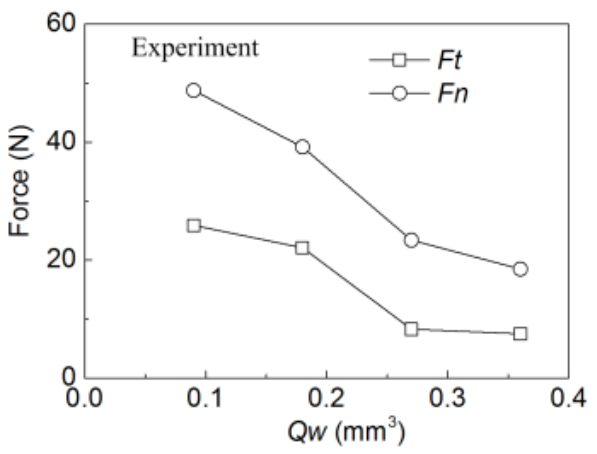

d

Fig. 9 The scratching forces of SPH simulation (a), experiment original force signals (b), smooth forces (c) and experiment force variation $(\mathrm{d})$ 
To simplify the scratching model, the stress distribution could be assumed as a cantilever beam [14] (Fig. 10). The scratching forces, which include impact force, holding force $F_{b o n d}$, scratching resistance, frictional force and chip-load, are acting directly on the SCD grit. These scratching forces, which produce a torque $M_{\mathrm{b}}$ to the cantilever beam, could be synthesized and measured by $F t$ and $F n$.

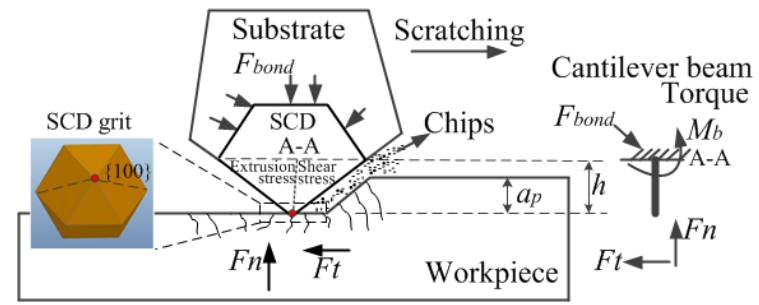

Fig. 10 The theoretical model of single SCD grit scratches on sapphire

The shear stress $\tau$ and extrusion stress $\sigma$ are principally produced by the $F t$ and $F n$ respectively. Since the SCD grit is an anisotropic crystal, the mechanical and physical property of each crystal plane is different. The $\{100\}$ and $\{111\}$ plane are oriented at the front and side of scratching direction respectively. The shear stress and extrusion stress are mainly maintained at the front and rear in scratching direction respectively [15]. However, once the shear stress or extrusion stress exceeds the strength of crystal planes, cleavage or fracture will appear in the SCD grit. The wear progress of SCD girt is principally determined by the stress state during the scratching process.

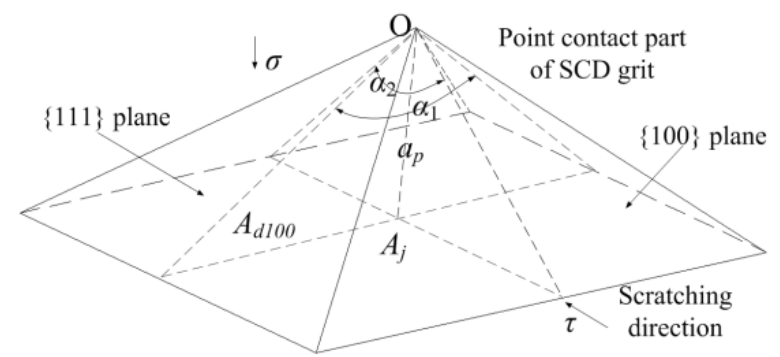

Fig. 11 Stress model of single SCD grit during scratching process

The scratching stress model of single SCD grit could be established as shown in Fig. 11. The $\alpha_{1}$ is the included angle of the two side plane $\{111\}$, and the $\alpha_{2}$ is the included angle of the two planes $\{100\}$. The $A_{d 100}$ is the projective plane area in the direction of shear stress. The $A_{j}$ is the projective plane area in the direction of extrusion stress. The $A_{j}$ and $A_{d 100}$ could be expressed as:

$$
\begin{aligned}
& A_{j}=4 a_{p}^{2} \tan \frac{\alpha_{1}}{2} \tan \frac{\alpha_{2}}{2}, \\
& A_{d 100}=a_{p}^{2} \tan \frac{\alpha_{1}}{2} .
\end{aligned}
$$

Therefore, the shear stress $\tau$ and extrusion stress $\sigma$ could be defined as:

$$
\tau=\frac{F t}{A_{d 100}}=\frac{F t}{a_{p}^{2} \tan \frac{\alpha_{1}}{2}},
$$

$$
\sigma=\frac{F n}{A_{j}}=\frac{F n}{4 a_{p}^{2} \tan \frac{\alpha_{1}}{2} \tan \frac{\alpha_{2}}{2}} .
$$

The size parameters of experimental SCD grit (Fig. 1, a) are calculated in the above equations, and the $\alpha_{1}$ and $\alpha_{2}$ are $109.4^{\circ}$ and $90^{\circ}$ respectively. The values of $F t$ and $F n$ are calculated with the scratching experiments.

Therefore, the shear stress $\tau$ and extrusion stress $\sigma$ could be calculated as $51.69 \mathrm{GPa}$ and $23.79 \mathrm{GPa}$ respectively. The calculated shear stress is nearly to the simulation shear stress $(51.97 \mathrm{GPa})$ of the deviating SPH particles (Fig. 5), and it also exceeds the shear strength of $\{100\}$ plane (51.339 GPa) [16].

The calculated extrusion stress exceeds the cleavage strength of $\{111\}$ plane, and it leads to the cleavage of \{111\} plane for SCD grit [17]. The above analysis presents that the result of the SPH simulation is close to the experiment and calculation result. The SPH simulation can well illustrate the wear progress of SCD grit in the scratching process.

Compared with the other grit-work piece contact form (face contact [15]), the SCD grit with point contact form has less work piece material removal volume and shorter scratching life. Because the shear stress and extrusion stress of point contact form are larger and more prone to exceed the strength of SCD grit. Different contact forms lead to different stress states of the SCD grit.

\section{Conclusions}

1. The SPH simulation results are in good agreement with the experiment results. The SPH simulation is able to illustrate the wear characteristics of SCD grit.

2. The scratching forces fluctuate in a certain range and decreases with the increasing of workpiece material removal volume. Different degrees of wear appear in both the front and rear of SCD grit.

3. The SCD grit mainly sustains the shear stress and extrusion stress during scratching process. The shear stress is larger than extrusion stress. The wear progress and wear form are principally determined by stress state.

4. The SCD grit with point contact form is prone to cleavage and fracture during the scratching process. Different contact forms result in different stress states.

\section{Acknowledgement}

The authors would like to acknowledge the support of National Natural Science Foundation of China (NSFC) (Grant No. 51805175), Natural Science Foundation of Fujian Province (Grant No. 2018J05146), China Postdoctoral Science Foundation (Grant No. 2019M652231), Fund of the Institute of Manufacturing Engineering of Huaqiao University (Grant No. 2018IME001), Young and Middle-aged Teacher Education Research Project of Fujian Province (Grant No. JAT201275) and Foundation of Doctoral Research Initiation of Zhangzhou Institute of Technology (Grant No. ZZYB2101).

\section{References}

1. Kim, H.; Manivannan, R.; Moon, D.; Xiong, H.; Park, J. 2013. Evaluation of double sided lapping us- 
ing a fixed abrasive pad for sapphire substrates, Wear 302(1-2): 1340-1344.

https://doi.org/10.1016/j.wear.2012.11.075.

2. Kong, C.; Liang, Z.; Zhang, D. 2017. Failure analysis and optimum structure design of PDC cutter, Mechanika 23(4): 567-573. https://doi.org/10.5755/j01.mech.23.4.14932.

3. Johnson, G. R.; Petersen, E. H.; Stryk, R. A. 1993. Incorporation of an SPH option in the EPIC code for a wide range of high veloeity, impact computation, International Journal Impact Engineering 14:385-394. https://doi.org/10.1016/0734-743X(93)90036-7.

4. Duan, N.; Yu, Y.; Wang, W.; Xu, X. 2017. SPH and FE coupled 3D simulation of monocrystal SiC scratching by single diamond grit, International Journal of Refractory Metals and Hard Materials 64: 279-293. https://doi.org/10.1016/j.ijrmhm.2016.09.016.

5. Yan, G.; Wu, Y.; Wang, H.; Zhao, D.; Lu, F. 2019. Research on mathematical model and cutting characteristics in machining of natural marble by diamond coated tool, Mechanika 25(2): 165-171. http://dx.doi.org/10.5755/j01.mech.25.2.23309.

6. Rüttimann, N.; Roethlin, M.; Buhl, S.; Wegener, K. 2013. Simulation of hexa-octahedral diamond grain cutting tests using the SPH method, Procedia CIRP 8: 322-327. https://doi.org/10.1016/j.procir.2013.06.110.

7. Varga, M.; Leroch, S.; Eder, S. J.; Ripoll, M. R. 2017. Meshless microscale simulation of wear mechanisms in scratch testing, Wear 376-377(2): 1122-1129. https://doi.org/10.1016/j.wear.2016.11.023.

8. Guo, X.; Wei, Y.; Jin, Z.; Guo, D.; Wang M. 2013. A numerical model for optical glass cutting based on SPH method, International Journal of Advanced Manufacturing Technology 68(5-8): 17-29. https://doi.org/10.1007/s00170-013-4919-8.

9. Liu, M. B.; Liu, G. R. 2010. Smoothed particle hydrodynamics (SPH): an overview and recent developments, Archives of Computational Methods in Engineering 17(1): 25-76. https://doi.org/10.1007/s11831-010-9040-7.

10. Johnson, G. R.; Petersen, E. H.; Stryk, R. A. 1993. Incorporation of an SPH option in the EPIC code for a wide range of high velocity, impact computation, International Journal of Impact Engineering 14(1-4): 385394.

https://doi.org/10.1016/0734-743X(93)90036-7.

11. Zhu, H.; Tessaroto, L. A.; Sabia, R.; Greenhut ,V. A.; Smith, M.; Niesz, D. E. 2004. Chemical mechanical polishing (CMP) anisotropy in sapphire, Applied Surface Science 236(1-4): 120-130. https://doi.org/10.1016/j.apsusc.2004.04.027.

12. Castaing, J.; Mu, Oz. A.; Gomez Garcia D.; Dominguez Rodriguez, A. 1997. Basal slip in sapphire $(\alpha-$ A12O3), Materials Science and Engineering: A 233(12): 121-125. https://doi.org/10.1016/S0921-5093(97)00056-7.

13. Goel, S.; Luo, X.; Reuben, R. L.; Pen, H. 2012. Influence of temperature and crystal orientation on tool wear during single point diamond turning of silicon, Wear 284-285: 65-72.

https://doi.org/10.1016/j.wear.2012.02.010.

14. Brookes, E. J.; Greenwood, P.; Xing, G. 1999. Friction and wear of synthetic diamond, International Journal of Refractory Metals and Hard Materials 17(1-3): 69-77.

https://doi.org/10.1016/S0263-4368(99)00012-8.

15. Wu, H. Y.; Huang, H.; Xu, X. P. 2018. The influence of crystallographic orientation on wear characteristics during single abrasive diamond grit scratching on sapphire, Industrial Lubrication and Tribology 70(8): 1414-1421. https://doi.org/10.1108/ILT-10-2017-0290.

16. Mikaelian, K. O. 2011. Testing diamond strength at high pressure, Diamond and Related Materials 20(10): 1340-1343. https://doi.org/10.1016/j.diamond.2011.09.005.

17. Wu, H. Y.; Huang, H. 2020. Influence of contact form on mechanical wear characteristics of single diamond scratching on Ta12W, Industrial Lubrication and Tribology 72(10): 1311-1316.

https://doi.org/10.1108/ILT-01-2020-0024.

H. Wu

\section{EXPERIMENT AND SMOOTH PARTICLE HYDRODYNAMICS SIMULATION FOR THE WEAR CHARACTERISTICS OF SINGLE DIAMOND GRIT SCRATCHING ON SAPPHIRE}

$\mathrm{S} \mathrm{u} \mathrm{m} \mathrm{m} \mathrm{a} \mathrm{r} \mathrm{y}$

Abrasive single crystal diamond (SCD) grit is widely used in the machining process of sapphire. The wear of SCD grit has a significant influence on the surface quality of sapphire. In this paper, smooth particle hydrodynamics (SPH) method is employed to reveal the wear mechanism of SCD grit with Steinberg constitutive equation and Grüneisen state equation. The wear morphology, wear volume and scratching forces are measured and analyzed by combination of SPH simulations and experiments. The results show that the scratching forces fluctuate in a certain range and decrease with the increasing of workpiece material removal volume. Different degrees of cleavage and fracture appear in the front and rear of SCD grit. The shear stress and extrusion stress are the main stresses of SCD grit during the scratching process. The wear progress and wear form are mainly determined by the stress state. Different stress state leads to different wear progress of the SCD grit. The SPH method is able to reflect and illustrate the wear characteristics of SCD grit scratching on sapphire.

Keywords: smooth particle hydrodynamics, single crystal diamond grit, scratching test, wear, sapphire.

Received July 21, 2020 Accepted June 02, 2021 